\title{
La educación del cuerpo en la comunidad Kichwa Mandari Panga. Cosmovisión de los pueblos originarios en el cuerpo
}

\author{
Body education in the Kichwa Mandari Panga community. Worldview of the indigenous peoples in the body.
}

Mariano Escobar

Universidad Nacional de La Plata, Facultad de Humanidades y Ciencias de la Educación, Argentina

marian_escobar31@hotmail.com

\begin{abstract}
Resumen:
El siguiente artículo propone describir y analizar la educación del cuerpo observada en la comunidad Kichwa Mandari Panga de la amazonía ecuatoriana, desde una mirada centrada en la recuperación de saberes de los pueblos originarios de América Latina. El mismo se basa en la información obtenida en el trabajo etnográfico llevado a cabo en la comunidad, en triangulación con distintos documentos de comunidades de pueblos originarios. A partir de esto, el trabajo pretende describir cómo las distintas representaciones del cuerpo en la comunidad, expresadas en las cosmovisiones kichwas, se encuentran ligadas a determinadas prácticas corporales. De esta manera, se plantea la posibilidad de comenzar a analizar a la educación del cuerpo de la comunidad desde sus saberes ancestrales en diálogo con los saberes de la Educación Física. Una educación del cuerpo que asume una íntima relación con el ambiente y con la comunidad, contrapuesta a los enfoques hegemónicos que consideran a las personas como individuos y al ambiente como un recurso natural.
\end{abstract}

Palabras Clave: Cuerpo, Educación, Pueblos originarios, Territorio.

\section{Abstract:}

The following article describes and analyzes the body education observed in the Kichwa Mandari Panga community of the Ecuadorian Amazon, from a perspective focused on the recovery of knowledge of the original peoples of Latin America. It is based on the information obtained in the ethnographic work carried out in the community, in triangulation with different documents from communities of indigenous peoples. From this, the work tries to describe how the different representations of the body in the community, expressed in the Kichwa worldviews, are linked to certain bodily practices. In this way, the possibility of beginning to analyze the community's body education from its ancestral knowledge in dialogue with the knowledge of Physical Education is raised; an education of the body that assumes an intimate relationship with the environment and with the community, opposed to hegemonic approaches that consider people as individuals and the environment as a natural resource.

KEYWORDS: Body, Education, Indigenous peoples, Territory.

\section{INTRODUCCIÓN.}

El presente artículo se basa en la investigación realizada en la comunidad Mandari Panga, en el marco de la realización de la tesina de grado de la licenciatura en Educación Física en el año 2015. Esta es una comunidad originaria Kichwa, ubicada en la Amazonía ecuatoriana, dentro del Parque y Reserva Natural Yasuní. En la misma, la práctica de la Educación Física en el contexto escolar es inexistente y por lo tanto dicha educación se da en diferentes espacios y a partir de otras formas de saber a las que plantea tal disciplina. Esto nos habilita a preguntarnos: ¿cómo se da esta educación? ¿bajo qué actividades o saberes? ¿qué resultados se obtienen?pero a su vez, también nos permite reflexionar sobre los saberes de la Educación Física: de qué manera pueden aplicarse tales saberes en dicho contexto, o si -a la inversa- se pueden tomar los saberes de la educación del cuerpo de esta comunidad y aplicarlos en la Educación Física.

Estas preguntas formaron parte de los interrogantes centrales a la hora de experimentar situaciones en campo, en las cuales el contexto cultural era muy diferente al propio (comunidades de pueblos originarios, afrodecendientes o rurales), y por lo tanto las categorías provenientes de la formación académica no parecían 
satisfacer estos interrogantes. Por esta razón surge la pregunta que orientó la investigación: ¿Cuál es el lugar de la Educación Física en las comunidades de pueblos originarios, en donde la educación del cuerpo no se lleva a cabo a partir de esta disciplina? Esta pregunta únicamente puede responderse una vez que comenzamos a comprender las culturas de los pueblos originarios y los procesos históricos por los cuales la cultura occidental ha negado sus saberes y su cultura.

De esta manera, la investigación se orienta en un primer momento, hacia la captación del significado del cuerpo para esta comunidad, para luego poder analizar su educación. En este marco, el objetivo de este artículo es avanzar en una descripción y análisis de una cosmovisión específica en relación al cuerpo en una comunidad originaria de América Latina. A su vez, derivado de ello, se busca problematizar algunos de los supuestos de la Educación Física desde perspectivas provenientes de los pueblos originarios.

Es así que este trabajo se inicia con el abordaje de una comunidad de pueblos originarios, que nos permitió, a partir del registro de sus experiencias corporales cotidianas, aproximarnos a comprender su visión del cuerpo y su educación. La manera de abordar esta investigación fue -en el sentido que podría plantear Wacquant (2006) - a partir y desde el cuerpo, considerándolo como objeto y vector de conocimiento. Por lo tanto, consideramos a la etnografía como una herramienta eficaz para corporizar el conocimiento. La descripción densa (Geertz, 1973) representó la principal técnica de recolección de información. La misma recurrió a la transcripción, en un cuaderno de notas de campo, de la totalidad de las experiencias que acontecían diariamente, la elaboración de descripciones detalladas en profundidad sobre prácticas, relaciones, reacciones, conversaciones, etc. con el fin de captar el sentido de las prácticas, intentando a la vez de sumergir al lector/ a de la más cercana posible en dichas experiencias y hacerlas inteligibles.

Ese registro se acompañó de entrevistas en profundidad, conversaciones informales, observaciones (participantes y no participantes), fotos, audios y videos. Cabe decir que estas últimas técnicas no fueron las utilizadas habitualmente, debido a su carácter "invasivo" y a fin de evitar interrumpir el flujo corriente de la vida cotidiana.

Las prácticas que se abordan con mayor detalle en este trabajo son, por un lado, aquellas que se encuentran relacionadas con la Educación Física. Y por otro, aquellas experiencias que permitan acercarnos a comprender a la visión del cuerpo de los pueblos originarios en América Latina, en donde se toma como punto clave, las interpretaciones posteriores al trabajo de campo, partiendo de las experiencias y relatos de los y las comuneros/as. Para las primeras, las descripciones están orientadas a prácticas en las cuales se evidencie el juego y el deporte. Para las segundas, la relación del cuerpo con las plantas medicinales, métodos de sanación del cuerpo, mitos en relación a la naturaleza comprendidos dentro de la cosmovisión Kichwa.

El recorrido que se propone es el siguiente: en la primera sección, se presentan algunas categorías teóricas que orientaron el análisis del caso; en la segunda sección, se presenta un resumen de la etnografía en la comunidad; en la tercera, a partir del análisis de estas dos secciones, se presentan algunas conclusiones en relación a las visiones del cuerpo de la comunidad. En la cuarta, analizaremos desde la Educación Física la relación entre los saberes del cuerpo analizados en la comunidad (desde su cosmovisión) y sus prácticas corporales, con el fin de indagar sobre su educación del cuerpo. Por último, el artículo se cierra con unas reflexiones finales en torno a la importancia del territorio (entendido como totalidad) para mantener y resignificar estos saberes y prácticas.

\section{Cuerpo, Cultura y educación. Algunos aportes Conceptuales}

La Educación Física se constituye en Argentina y en otros países de Latinoamérica a comienzos del siglo $\mathrm{XX}$, como disciplina encargada de educar a partir del cuerpo en la institución escolar. Ésta, se ve ligada a lo largo de su historia, a determinados sentidos políticos que configuran sus modos de acción, inclusive en la actualidad. De este modo, en Latinoamérica se da un proceso de implantación de un proyecto europeo, que entiende la heterogeneidad propia de nuestras tierras enmarcada en la dicotomía civilización-barbarie. Así, 
Sarmiento en 1886, toma a la gimnástica como un recurso significativo para la difusión de los valores nacionales y símbolos patrios, con el fin de homogenizar el crisol de razas del naciente estado nacional, considerando así, que la gimnástica civilizaría a los Tobas (Galak; 2014).

Sin embargo,a pesar de los diferentes sentidos políticos que carga nuestra disciplina en el devenir histórico, debemos considerar también, que la educación del cuerpo, o de los cuerpos, es previa al nacimiento de la Educación Física como disciplina escolar. Galak (2014) lo denomina como educación física (con minúsculas), a aquellas actividades corporales ligadas a la transmisión de saberes, como puede ser desde una técnica de carrera o lanzamiento, hasta el control de los esfínteres, por ejemplo. De esta manera, el análisis de la educación del cuerpo en la comunidad estudiada, está ligado a la transmisión de sentidos que hay en sus prácticas corporales. Es desde allí, donde la Educación Física construye sus saberes; en sus prácticas (Crisorio y Bratch; 2003).

Para esto, es pertinente tener en cuenta los marcos epistemológicos en que construimos nuestros saberes y sentidos en relación a tales prácticas, siendo estos determinantes a la hora de comprender las visiones del cuerpo y la educación en el contexto estudiado. Tenemos en cuenta entonces que, además de construir el conocimiento desde las propias prácticas, en la gran mayoría de facultades de América Latina, en las carreras de Educación Física el conocimiento proviene de diversas disciplinas (fisiología, anatomía, sociología, filosofía, antropología, ciencias de la educación, etc); compartiendo mayoritariamente, enfoques provenientes de países europeos o estadounidenses. Este tipo de planteamiento, supone entender que estos autores del Norte pensaron y piensan tales categorías desde su realidad, su mundo en términos de Heidegger (Dussel, 1994), desde sus experiencias. Por ello, estas perspectivas presentan limitaciones para comprender algunos conceptos o categorías que se dan en otros contextos socioculturales, pertenecientes a otros marcos epistemológicos, como los que queremos analizar. Esto no significa renunciar a sus aportes, sino que, en todo caso, deben ser incorporados al diálogo junto con otras formas de saber (Boaventura De Sousa; 2009).

Para mencionar algunos ejemplos que sirvieron de punto de partida para realizar este trabajo, podemos hablar del poder que se ejerce sobre los cuerpos en la modernidad a partir de la disciplina y la biopolítica en el caso Foucault (2009); distintas perspectivas sociológicas que coinciden en abordar el poder enclasante que se ejerce sobre los cuerpos en las instituciones educativas de la modernidad (Bourdieu y Passeron, 1996, Willis, 1988, entre otros). Asimismo, autores locales como Carballo (2003), Crisorio y Bratch (2003), entre otros, lograron articular estos saberes del cuerpo para construir una Educación Física que se desliga de una educación basada en el cuerpo orgánico, individual, que plantean las ciencias biológicas. En suma, estos trabajos sientan las bases para analizar y ver al cuerpo como un fenómeno social y cultural, revisando la visión hegemónica del cuerpo/ organismo. Asimismo, dan cuenta de instancias de modelación social de los cuerpos más allá de las prescripciones de las instituciones educativas estatales, como son los casos de los trabajos realizados por Descola (2012), Leenhart (1997), Lambeck (2010).

Autores/as como Dussel (1994), Rivera Cusicanqui (2010), Kushc (2015), Segato (2016), nos ayudan a comprender que la manera de entender la transmisión de sentidos de los pueblos originarios, es a partir de otras formas de conocimiento, que difieren del conocimiento científico. Ante esto, coincidimos con Dussel (1994, 2007), al destacar que los saberes de los pueblos originarios se encuentran traducidos en formas de mitos. Estos son relatos racionales en base a símbolos que dan sentido a la vida, y que pueden ser reinterpretados a lo largo de la historia, expresando nuevas configuraciones.

\section{Contexto etnográfico}

Mandari Panga es una comunidad Kichwa ubicada en la amazonia ecuatoriana, perteneciente a la Parroquia Dayuma, Cantón Francisco de Orellana, Provincia de Orellana. Contiene 6.255,32 Has, de las cuales el 50\%está dentro del Parque Nacional Yasuní, área protegida de importancia nacional y mundial. Sus pobladores están dedicados principalmente a actividades como la caza, la pesca y la recolección de frutos de la chacra 
para su subsistencia. La principal fuente de ingreso es la agricultura, a través de la producción de la yuca, chonta, hojas del bijao y el plátano, que son comercializados en la ciudad del Coca, (ciudad más cercana a la comunidad) la cual se encuentra a hora y media de navegación por el rio Tiputini y dos horas en colectivo desde la ruta terrestre más próxima. Los primeros fundadores de Mandari Panga provienen de la comunidad de Satzayaku, la parroquia Canelos en la Provincia de Pastaza y, parroquia Pano en la Provincia de Napo. Pertenecen al grupo étnico de los Naporunas, quienes se han asentado en las riberas del río Napo y el Tiputini desde tiempos ancestrales.

En estudios como el de Ortiz (2012) y Viteri Gualinga (2005), se puede advertir que actualmente los pueblos kichwas de Ecuador, se encuentran unidos bajo diferentes organizaciones en Pastaza cuya finalidad es consolidar las circunscripciones Territoriales Indígenas, mediante la construcción de una propuesta política de territorio autónomo. Dicha propuesta está basada en la consolidación de un territorio con sus propias formas y políticas de organización social, económica, cultural, ambiental, tecnológica y de recursos humanos, con el fin de lograr la vigencia del Sumak Kawsay (vida en armonía), el Sumak Allpa (tierra sin mal, ligado a la tenencia y uso de ésta) y el Sumak Yachay (ligado a los saberes ancestrales). Para los kichwas, el control significa que las familias de una comunidad en particular y del Pueblo Kichwa en general puedan decidir sobre su territorio y el manejo autónomo de sus recursos, ejerciendo sus propios sistemas económicos, sociales y culturales. De este modo, se plantean una nueva organización social basada en el fortalecimiento de los lazos familiares en el Ayllu (familia extensa) a través del cual las personas tienen derecho a cazar, pescar, recolectar y cultivar en el territorio comunitario; así como el fortalecimiento de las relaciones entre familias y comunidades, basadas en sus saberes ancestrales o Sumak Yachay.

El encuentro con esta comunidad, no fue programado. Entre diciembre del 2014 y marzo del 2015, en el marco de un viaje personal como viajero independiente por algunos países de América del Sur, procuro buscar una comunidad de pueblos originarios que no cuente con la disciplina de la Educación Física, con el fin de observar e indagar su tipo de educación del cuerpo. Así, llego a la ciudad de El Coca en Ecuador, sabiendo de antemano que a sus alrededores existen diversas comunidades de pueblos originarios. Comienzo a preguntar a la gente que se encuentra cerca del río Napo sobre la posibilidad de visitar alguna comunidad, pero la mayoría de veces me envían a las agencias turísticas. Estas se caracterizan generalmente por transmitir una visión folklórica de estos pueblos, donde se busca resaltar parte de la cultura que el turista desea ver y comprar. De esta manera, basándome en experiencias anteriores, se hace imposible intentar dialogar abiertamente o realizar actividades fuera de los planes, ya que el principal motivo es comercial.

Sin embargo, cerca del rio se encuentra la federación Kichwa, donde casualmente ingreso y sigo preguntando, ya más en planes de buscar un lugar para dormir por esos lugares y descansar antes que comience la lluvia amazónica. Allí hablo con dos hombres Pedro Mamallacta y Matías Dawa, que muy sonrientemente me dicen que son kichwas y que puedo ir a convivir con ellos, siempre y cuando la presidenta de la comuna quiera. Entre ellos siempre hablan en kichwa, pero conmigo en castellano. Gran parte de la comunidad se encuentra en la federación por cuestiones burocráticas, relacionado a temas de medio ambiente. Me presentan a la presidenta y la gente que estaba cerca de ella. Converso con una señora mayor, llamada Damiana, que es la fundadora de la comunidad y seriamente me dice que puedo ir a su casa y también a la de Matías y Pedro, que me van a llevar a conocer la comunidad y enseñarme su cultura. Solo llevo algunos alimentos que faltan para comer, por ejemplo arroz, que se encuentra fuera de época, pero después, como me dice Pedro; "comida allá abunda, hay de todo".

Termina la reunión programada y la presidenta me presenta ante las personas presentes compartiéndome su bebida ancestral; la chicha de yuca. Me explica que para cada celebración, después de una reunión o para invitar a alguien de afuera, lo primero que se comparte es la chicha, que no solo calma la sed, sino que también alimenta y en grandes cantidades embriaga. Esta bebida puede beberla hasta un bebé lactante, como forma de alimento, ya que comentan que cuando no hay comida, sólo se bebe chicha y así el hambre pasa. 
La gente a simple vista, viste de la misma forma que en la de la ciudad, principalmente los/as jóvenes, con relojes, celulares, aunque también algún que otro colgante de dientes o semillas brillosas, que pueden llegar a evidenciar algún tipo de artesanías propias de la cultura Kichwa y/o amazónica. Pero muy desligados del estereotipo que se ve en los carteles de las empresas turísticas. También algunas mujeres mayores visten ropa tradicional, la cual es una camisa larga con varios "dibujitos" pequeños y coloridos.

Converso un buen rato mientras seguimos tomando chicha, y las charlas son muy fluidas, intercambiando que hay acá y que hay allá de los distintos países. Como buen extranjero, en realidad soy más entrevistado que entrevistador. A las pocas horas, tomamos un bus y vamos hacia el rio Tiputini donde nos espera una canoa a motor. Allí me dice Damiana, la fundadora, de 72 años, a la cual la gente le tiene mucho respeto y le dicen la abuelita, que me voy a quedar los primeros días en casa de Dawa y después voy a ir con ella, que me quiere enseñar cómo viven en la finca. Y cuando alguna canoa vuelva a salir de la comunidad es cuando puedo regresar.

Llegamos de noche a la casa de Dawa y descansamos en las hamacas inmediatamente. Cuando decido levantarme ya es de día, madrugada. Salgo de la casa y puedo ver todo con claridad, luego de un intenso aguacero. Los sonidos de la selva se llevan el lugar principal de mi atención. Decenas de pájaros emitiendo sonidos diversos, e insectos imposibles de identificar bajo un constante fluir de agua proveniente del rio. Quedo perplejo y comienzo a observar todo con detalle, estando a menos de un metro de la casa, descalzo sobre el húmedo pasto, sintiendo el calor con lo poco que llevo puesto; una camisa finita y una malla gastada.

Los días con Dawa y Pedro me sirvieron principalmente para reconocer gran parte de lo que nos brinda el medio ambiente para vivir en ese territorio. Por las mañanas ya bien temprano, a eso de las 5, Dawa ya se encuentra desde hace rato bebiendo su té de guayusa, una bebida de la hoja de esa planta, la cual me comentan que tiene propiedades diversas, como dar ánimo, energía, calma dolores corporales, es buena para el dolor de cabeza y panza, entre otras. Además, es una planta que sus antepasados la utilizaban para ir a cazar, bebiéndola desde las 2 am para adquirir su fuerza y tener éxito en la cacería. Esto, combinado con ponerse ají en los ojos, simbolizaba una mayor eficacia en la cacería, ya que el jaguar así no puede detectarlos. Hoy en día generalmente solo se bebe guayusa.

Luego de tomar el té, bien tranquilo al lado del fuego, desde las últimas horas de la oscuridad de la noche hasta que amanece y un ratito más, Dawa sale a revisar las redes que deja la noche anterior, previamente bañándose en el rio a pocos metros de su casa en el rio Tiputini, para ablandar el cuerpo. Así dice que luego de beber la guayusa y bañarse, da ese efecto de aflojamiento corporal y se siente más fuerte. Lo que compruebo instantáneamente con mi propio cuerpo. Lo acompaño en su pequeña canoa, que él mismo hizo, y como de costumbre sacamos entre 3 y 7 pescados, que preparamos inmediatamente para el desayuno, ya a eso de las 6 o 7. Es costumbre acompañar el pescado con yuca, plátano, chonta, cocidas en sopa, o asadas, fritas, y con una gran variedad de frutas, que también se obtienen a pocos metros de la casa, como por ejemplo: papaya, mango, banana, piña, guabas, chirimolla, maracuya, uvas, entre muchas otras.

Al conocer mis intenciones de aprender sobre el conocimiento del cuerpo que ellos tienen, lo primero que se les ocurre es invitarme a dar un paseo, no muy lejos de su casa, por el monte. Allí, mientras caminamos muy tranquilamente, me van explicando los usos medicinales de cada planta, los cuales varían desde un dolor de panza hasta por ejemplo reducir un cáncer. Me dicen que es su farmacia natural, que entre ellos mismos se curan, por ejemplo, echando hojas de mentol en una inflamación y frotando las manos para dar calor, preparando tés para mejor funcionamiento de los riñones y otros órganos, baños de vapor para problemas en la piel, etc. Muy pocas veces han ido al médico y dicen que sus abuelos, sacaban todo del monte para cuidar su cuerpo, porque sabían más que ellos. Pero me comentan, que no solo es saber qué hace cada planta, sino que también uno debe saber conectarse con el espíritu de cada una de ellas. Porque cada planta tiene un dueño. Aunque también los animales lo tienen y todo lo perteneciente a la naturaleza. Todo tiene vida en la naturaleza. De allí se desprenden varias historias en relación a la creación del mundo, cómo conseguir pareja, 
la buena o mala suerte, los antepasados, etc. Conversaciones que principalmente se dan alrededor del fuego de noche, escuchando los sonidos nocturnos de los animales y el ambiente.

Sobre este tipo de charlas en relación a los espíritus, surgen otros temas como los de los chamanes. Ellos son los sabios del cuerpo, de protegernos, pero así también de hacer daño. Esta forma de curar o de perjudicar a alguien, tiene que ver con la envidia, los malos pensamientos, las malas acciones contra otras personas, etc. Quiere decir que, a través de los espíritus de las plantas, los chamanes pueden sacar o poner el mal dentro del cuerpo de las personas. Porque son los que mejores se conectan con estos, son los que pueden sentir toda la naturaleza, por eso viven más aislados (algunos), hacen rituales de ayahuasca (donde pueden ver lo que le pasa a la persona por dentro), llevan una dieta estricta que les permite tener estas visiones, son personas mayores (varones o mujeres) donde a mayor edad, mayor saber. Ellos mismos se han curado varias enfermedades corporales con ellos, y son de su predilecta confianza. Muchas formas de curar, tienen que ver con la ingesta de algún té, aplicación de especie de crema o polvo sobre la zona afectada, realizar una determinada dieta de alimentos, etc., pero otras, que me resultaron más sorprendentes, son las que están relacionadas con las oraciones, cantos, soplar humo, colocar plantas en un objeto determinado, etc. Acciones que no necesariamente necesitan la presencia del cuerpo del paciente para sanarlo, sino que se puede hacer desde otra representación, a distancia.

En cuanto a los trabajos corporales que realizamos cotidianamente, destacamos principalmente la pesca y la agricultura. A su vez tenemos mucho tiempo de ocio para charlar e intercambiar formas de vida preguntándonos de todo, aunque en los momentos de trabajo, también lo hacemos conversando y tranquilos, sin un tiempo determinado, ni apuro. El cultivo y cosecha de yuca y plátano son las más importantes, aunque como en todas las actividades, ellos tratan de no hacerme trabajar mucho o, mejor dicho, prácticamente muy poco, demostrándome constantemente que quieren que la esté pasando bien. Es por eso que cada vez que nos transportamos por el rio en canoa para ir a visitar a alguien o al centro comunal, no me dejan remar mucho porque dicen que me voy a cansar, lo que resulta verdadero, ya que requiere de un enorme esfuerzo mantener el remo por más de media hora seguida. Su aspecto físico a simple vista, demuestra rasgos de excelente salud, siendo personas mayores muy activas y hábiles. Por eso ellos mismos, afirman que son muy sanos, porque trabajan desde niños, comen todo natural y en abundancia. Me lo demuestran constantemente, como por ejemplo Dawa que es ya mayor de 70 años, sube con facilidad a una palma para bajar un fruto. Estas actividades las fueron aprendiendo de las personas mayores, imitándolas, pero también haciéndoles caso, ayudando en las mingas (trabajo comunitario).

Sobre sus creencias religiosas, ellos aseguran ser evangélicos. Al conversar del tema, les pregunto cómo se dice Dios en kichwa. Ellos me responden: Inti iaia, que significa padre sol. También me cuentan que hay que dar gracias a Dios por todo lo que nos da la naturaleza, por eso su inmenso respeto y cuidado, nunca deben tomar de más, sólo lo necesario, para poder conservarla, por eso le deben el mayor respeto, ya que sin ella (refiriéndose en términos de pachamama) no viven, no comen, no construyen sus casas, medicinas, transporte, etc. Así les enseñaron a vivir sus ancestros, tomando lo necesario y tratando bien al ambiente para que se regenere:

- "Aqui hay de todo, tanto que sobra, por eso lo tenemos que tirar o se lo damos a las gallinas, por eso están tan gordas, se alimentan bien. Si vas a la ciudad, hasta el agua tienes que pagar"

Luego de 7 días, voy a vivir a la casa de Damiana. Me llevan Dawa y Pedro en canoa, aproximadamente dos horas de viaje, y como de costumbre, a medida que viajamos, me siguen contando los beneficios corporales de tomar alguna planta o caldo de animales. Damiana y su familia, en la cual hay varios niños/as, jóvenes adolescentes y algunas personas mayores, nos reciben enseguida con chicha de yuca. Totalmente necesaria, luego del intenso calor y para recuperar energías. En esta familia, no todos comparten entres sí lazos sanguíneos, aunque la gran mayoría sí. Viven allí una hija de Damiana con sus hijos pequeños, un adolescente nieto de ella y dos hombres jóvenes amigos de la familia. Mi casa es así dice Damiana, la llamada abuelita, 
"aqui cualquiera puede veniry si se comporta bien, y es respetuoso se puede quedar, siempre van y vienen personas, pero todos tenemos que trabajar, vagos no queremos".

De esta manera me explica seriamente que ella me va a enseñar a trabajar, usar machete, cultivar, desmontar, etc., porque esa es la manera de vivir aquí, y para comer se debe trabajar, no se puede comprar la comida como en la ciudad. Así me explica que ella no come comida de la ciudad, o muy pocas veces, porque dice que tiene químicos, y ella puede producir toda su comida, que es bien sana porque la planta ella misma, sin químicos y natural. Este fue uno de los motivos por los cuales Damiana decide volver a su lugar de origen, la finca, ya que, antes de estar en Mandari Panga, vivió varios años en la ciudad, donde su tipo de vida fue muy diferente.

Es así que esos días con la familia, trabajamos bastante, aunque no me resulta tan agotador, en el sentido que siempre lo hacemos en conjunto, entre risas y charlas, y también, ¿por qué no?, a veces: jugando. Desmontamos parte del monte con machete para preparar el terreno para cultivar yuca, cargamos racimos de plátanos y chonta para llevar a vender a la ciudad, juntamos agua del rio para cocinar, cortamos el pasto con machete cerca de la casa para ahuyentar insectos y animales, buscamos mates y fibras naturales para hacer artesanías, pelamos maíz, cacao, etc. Ante esto, conversamos durante la noche, antes de dormir, que estos trabajos siempre se hacen en conjunto, con la familia y comúnmente en mingas. Esta es la manera que sus antepasados le enseñaron a trabajar. Por ejemplo, si Damiana necesita desmontar un terreno grande para cultivar yuca posteriormente, ella convoca a otras familias para que le ayuden, así el trabajo se hace rápido, en un día. Esto es igual para construir una casa o cualquier otra actividad. Participa en ella toda la familia, desde el niño/a más pequeño/a hasta los/as ancianos/as, cumpliendo diferentes roles. La forma de "pago" es con el almuerzo y con chicha durante las horas de trabajo. Si hay, también cena, sino más chicha. Sin chicha no hay minga dicen algunos sonriendo. A su vez, las personas que fueron a trabajar a lo de Damiana, cuando ellos/ as necesiten de su ayuda para su minga, ésta debe presentarse, devolviendo así el trabajo.

Todos los días al finalizar el día a eso de las $5 \mathrm{pm}$, bebemos chicha, que nos calma la sed, alimenta y relaja. Es muy gratificante beberla luego de un día a puro sol y en constante movimiento, aunque durante el trabajo también se toma, ya que es muy raro que en esta cultura se tome agua para calmar la sed. Las mujeres son las encargadas de cultivar, cosechar y preparar esta bebida, raramente los hombres se encargan de esto, por lo que en la casa de Dawa, al no haber mujeres no hay chicha. A su vez, me comentan una tradición; "si usted o cualquiera que va a la casa de una familia y rechaza la chicha, esa mujer no le va a dar más de comer en todo el dia, puede estar abi muriéndose de hambre que no le van a dar". Por suerte nunca se me ocurrió rechazarla. Las mujeres en este caso, son las que sirven la bebida, pero a su vez elijen a quién y cuantas veces repetirle.

Las personas mayores, o a veces los mismos niños, que me acompañan en los trabajos, se encargan de explicarme bien la tarea, cómo manejar las herramientas, demostrándomelo varias veces hasta que me salga, o al menos un poco, ya que mis habilidades corporales para estas tareas, no son tan efectivas como cuando lo realizan ellos/as. Al igual que Dawa y Pedro, se evidencian grandes habilidades a la hora de trabajar y moverse. Damiana que es la mayor dice que ella es bien sana porque es la que más trabaja, y así da el ejemplo a la gente menor. Porque comida hay de sobra, solo hay que trabajar.

Mientras pelamos cacao, con uno de los hombres que vive temporalmente en la casa de Damiana, conversamos sobre mi interés en conocer sobre saberes del cuerpo en la cultura Kichwa. Éste me comenta lo anteriormente mencionado por Dawa, sobre los espíritus de las plantas en la sanación del cuerpo y el saber de los chamanes sobre esto, agregando que la sanación o la enfermedad no dependen únicamente de estos factores, sino que es fundamental que la persona afectada este convencida de que quiere sanarse, sino no tiene sentido tal intervención y es muy difícil que lo logre, a pesar de la buena mano del curandero/a. Estas creencias en los poderes de los curanderos, comentan que son cada vez menos creíbles, por el abuso en los costes económicos y charlatanerías, en muchos casos. Sin embargo, no se duda que esta ha sido la forma de relacionarse sanamente con el cuerpo, sin la necesidad de hospitales y gente de afuera de la comunidad, por lo tanto, lo consideran algo digno de recuperar y de no perder del todo. 
Lamentablemente, luego de 4 días con esta hospitalaria familia, que me acepto abiertamente sin conocerme, viene una canoa del ministerio de medio ambiente, donde Damiana y la familia, aprovechan para regresar a la ciudad y vender algunas chontas y cañas de azúcar, por lo que debo regresar con ellas. Solo estuve 11 días en la comunidad, pero esta breve visita, hizo generar muy buenos lazos con las familias y motivación para seguir investigando en comunidades de pueblos originarios. Así continué visitando, a los pocos meses, otras comunidades, y a los tres años, regresé a Mandarí Panga dos meses y medio, viaje que duró 1 año y 4 meses recorriendo más de 12 comunidades de pueblos originarios de América del Sur, desde Argentina hasta Colombia.

\section{Reflexiones en torno a las representaciones del cuerpo en Mandari Panga.}

Para este tipo de comunidad que estudiamos, donde la forma de vida difiere ampliamente al estilo de vida en las ciudades u otros contextos culturales, partimos de la hipótesis de que la educación del cuerpo debe estar ligada a sus formas de saber y sus formas de vida, con el fin de construir y comprender una educación aplicada a las realidades locales. Esto nos lleva, por un lado, a indagar sobre la visión del cuerpo existente en la comunidad, con el fin de acercarnos a comprender su cultura y sus transmisiones de sentidos. En ésta, aunque el discurso hegemónico del cuerpo esté presente, pudimos identificar otras visiones diferentes, transmitidas de padres a hijos, a lo largo de las generaciones ${ }^{1}$. Esto nos ha posibilitado problematizar al cuerpo desde un lugar contrapuesto al discurso moderno- occidental que divide cuerpo y alma, y, por lo tanto, a partir de esto, a replantear el tipo de educación.

Entendemos así, que este discurso dualista es incorporado en América a través de un proceso que negó y niega a las culturas de pueblos originarios y por lo tanto sus saberes, lo que Grosfogel (2013) denomina como epistemicidios. Esta visión que ve al cuerpo como accesoria máquina al servicio del espíritu racional, no solo deja de lado la parte carnal (el cuerpo), sino que a su vez la afectividad, el amor, la voluntad, etc. Y por lo tanto justificó el genocidio contra los indígenas en América, al considerar que éstos no poseían alma (Dussel, 2007). Autores/as como, Descola (2015), Segato (2016), Dussel (2007) entre otros afirman que, en América Latina, antes de la llegada de los españoles, ya existían visiones del cuerpo, diferentes a la versión antropológica occidental del ser humano: dualismo alma - cuerpo. Por el contrario, los pueblos originarios parten de una visión total del ser humano, como un todo, el cual forma parte de la naturaleza (entendida como totalidad/ Cosmos, según Dussel; 1994). Podríamos decir entonces que estos pueblos ven al cuerpo como una unidad. Martínez Sarasola (2014) reflexiona críticamente, al estudiar a pueblos originarios de Argentina, afirmando que el "hombre" occidental se ha separado de la naturaleza, y al separarse, quiere dominarla. En cambio, el indígena se considera parte de la naturaleza y por lo tanto no busca dominarla, sino conservarla y respetarla.

Investigaciones como estas y otras, (López Austin, 1967; Citro, 2009; Huanacuni Mamani, 2010; Rivera Cusicanqui, 2010; entre otras/os) en pueblos originarios de América Latina, son las que nos posibilitan diferentes análisis de la visión del cuerpo en la comunidad estudiada. Esta misma, coincide con cosmovisiones de otros pueblos, la forma de vida comunitaria, y la relación con el ambiente, entre otros aspectos. Esto nos permite dar sentido a las observaciones realizadas en el campo. Entre las más importantes en relación a la visión del cuerpo, destacamos:

- La relación de las plantas, animales y otros seres considerados con vida, con los espíritus y los ancestros.

- La sanación del cuerpo a través de estos espíritus y el poder de los chamanes.

- La relación continua con el ambiente/ naturaleza.

Los primeros significantes del cuerpo que fueron apareciendo en la comunidad durante mi estadía, estaban vinculados a la mención de ciertos espíritus presentes en plantas y animales. Las plantas, animales y también 
otros seres vivos de la naturaleza poseen espíritus que interactúan con nosotros. Estos pueden vernos, cuidarnos y hasta dañarnos. A su vez, pueden mutar de un cuerpo a otro. Para este caso, los ancestros, pueden estar presentes en uno de estos seres, y a través de ellos nos podemos comunicar. Los chamanes, son los que pueden comunicarse con mayor facilidad con ellos, debido a que tienen el poder, para sentir la totalidad de la naturaleza. A través de la comunicación con estos espíritus, pueden sanar o hacer daño a una persona, sin la necesidad de su presencia física.

Las prácticas de la vida cotidiana en la comunidad, están íntimamente vinculadas al trato con el medio natural: cazar, pescar, preparar la chacra, cultivar, recolectar frutos y medicinas, elaborar herramientas y viviendas, recorrer grandes hectáreas a pie o canoa, etc. La gran mayoría de estas actividades se realizan en comunidad, en las llamadas mingas, o trabajo comunitario. En ellas participan tanto niños como adultos y ancianos, varones y mujeres. Donde los/as menos expertos/as en la actividad cumplen roles de menor complejidad, y van aprendiendo a partir de la observación de la gente más experta, que les explica y demuestra constantemente la práctica.

Estas observaciones pueden aportarnos diferentes sentidos para comenzar a comprender las visiones del cuerpo en estos pueblos a partir del siguiente análisis hermenéutico, pudiendo así interpretar distintos símbolos:

La naturaleza al estar cargada de espíritus y seres con vida, que a su vez representan a sus ancestros, como un todo viviente, es el motivo por el cual se le debe tanto respeto. Si esta es dañada o desaparece, lo mismo le ocurre al cuerpo "individual". De ella, se obtiene todo lo necesario para vivir. Por ejemplo, las medicinas, que son mayormente utilizadas por los chamanes o curanderos, dotan de poder a éstos gracias a su buena comunicación con los espíritus. Gracias a los análisis de Kusch (2015) y de los relatos de los/as comuneros/ as podemos entender, que su forma de saber se centra en esta comunicación. Dice el autor que el órgano predilecto del chaman en las sociedades andinas (raíz cultural de nuestra comunidad estudiada), es el corazón o sonko en quechua, el cual les permite sentir la totalidad de la naturaleza, contraria a la forma de saber, racional que surge en la modernidad occidental que toma al cerebro como referente de conocimiento.

Por lo tanto, el cuerpo, para estos casos, no puede ser analizado desde las nociones de las ciencias biológicas, únicamente como organismo. Debido a que este puede variar en distintos espacios y tiempos, como por ejemplo en una sanación, donde no se necesita obligatoriamente de la presencia física del "paciente". En esta cosmovisión, todo lo que muere, renace, se transforma. Esto determina a su vez, el tipo de trato con el ambiente. Basada en la convivencia comunitaria que toma de éste lo necesario para vivir, sin dañarlo, con el objetivo de que perdure. Este principio ético, político, económico y social es visto desde diferentes cosmovisiones en América Latina como el Buen Vivir que, aunque no involucre a la totalidad de pueblos originarios, sí es un principio, que según Huanacuni Mamani (2010) está vinculado a las demás cosmovisiones del continente (desde las mapuches hasta las maya), compartiendo esta visión del trato con la naturaleza.

\section{La educación del cuerpo en Mandari Panga desde la Educación Física.}

Teniendo en cuenta que, en las prácticas corporales cotidianas se transmiten los sentidos más importantes de su cultura que, a su vez, les permiten convivir con su ambiente desde sus propios saberes, provenientes de sus ancestros, a través de los relatos míticos que estructuran una determinada visión del mundo; es desde aquí que consideramos pertinente abordar a su educación del cuerpo.

Como vimos anteriormente, esta comunidad no cuenta con la disciplina de la Educación Física en su curricula, sin embargo, hay suficientes motivos que nos determinan que la educación del cuerpo en esta comunidad puede darse de otras formas y en otros espacios. Podemos concluir entonces que esta educación de la que hablamos, se da en diferentes espacios como la chacra, el monte, el rio, etc. en comunidad, y a partir de actividades corporales cotidianas. Por lo tanto, como pudimos observar, los/as niños/as o las personas 
menos "expertas", en las actividades cotidianas, que involucran la minga (trabajo comunitario), copian las actividades corporales de los más "expertos” y a su vez éstos/as les enseñan cómo realizarlas efectivamente.

Las formas de aprendizajes para las actividades de la vida cotidiana que les permiten desenvolverse en su cultura, se dan, a partir de lo que en Educación Física llamamos, como juego. Esta consideración se debe a que el juego es visto como una práctica en sí misma, donde el proceso cobra mayor importancia que el resultado (Pavía, 2006). Por lo tanto, los/as niños/as “juegan” desde muy tempranas edades, a las actividades que realizan sus mayores, probando nuevas herramientas, reconociendo los diferentes ambientes, técnicas, habilidades, entre otras; como por ejemplo, cazar, pescar, cosechar, preparar el terreno de cultivo, rozar, talar, recolectar, elaborar herramientas, artesanías, etc. Entonces, desde esta interpretación, estas actividades son elaboradas con fines en sí mismos, sin buscar resultados concretos, sino por el disfrute de la práctica. Por ejemplo; una niña de 2 años golpeando con un pequeño machete la tierra, o grupos de niños/as que salen a pescar donde el fin principal es divertirse y no la cantidad de pescados que traen, un niño arrastrando un bidón cortado a través de un hilo, cargado de malezas, etc.

Estas actividades, a medida que requieren mayores resultados, como sería la obtención del alimento para sobrevivir, son consideradas en nuestra cultura como trabajos. Ya no importa tanto la práctica en sí, sino el resultado. Sin embargo, algo notorio que debemos destacar, es que este pasaje que aquí intentamos explicar, no se da de manera total, sino que va tomando diferentes matices según los momentos y necesidades que se presentan en el tiempo. Esto se debe a que en las personas mayores también se pudo observar situaciones de trabajos cotidianos vinculados al juego. Ya que el disfrute por la actividad en ciertos momentos, prioriza sobre el resultado. Por ejemplo, ir a pescar o cazar, sin la necesidad rigurosa de obtener algo a cambio, más que el disfrute de la propia actividad. Podemos concluir entonces, que este vínculo entre el juego y el trabajo que analizamos, es uno de los aspectos más importantes que nos permiten interpretar y comprender su educación del cuerpo. Debido a que en estas prácticas corporales, se enseña, además de una técnica o trabajo corporal, la transmisión de los saberes culturales de su comunidad, los cuales están cargados de sentidos, que les permiten relacionarse con su entorno a partir de sus saberes y cosmovisiones.

Por otro lado, no debemos dejar de considerar, que todas estas prácticas, al estar en un mundo ya globalizado, las personas, la comunidad, va adoptando otros tipos de sentidos diferentes a los transmitidos de sus tradiciones provenientes de sus ancestros; esta es la característica fundamental de las culturas, la capacidad de transformarse continuamente (Garcia Canclini, 1990). Sin embargo, debemos ser rigurosos a la hora construir y transmitir esos "nuevos" sentidos que emergen en el devenir contemporáneo. Otras de las prácticas corporales que la Educación Física distingue como contenido educativo para el cuerpo, además de los juegos, son los deportes. Estos, forman parte de las prácticas corporales de los/as comuneros y comuneras de Mandari Panga. Principalmente el Voleybol y el Fútbol. Para su análisis, debemos considerar que, como toda práctica, se encuentra cargada de sentidos, los cuales son construidos históricamente y que pueden ir reconstruyéndose por los actores de acuerdo a su mundo cultural. También entendemos que, al ser una práctica moderna, históricamente carga ciertos sentidos de esta cultura. Para mencionar brevemente algunos de ellos, podemos referirnos a Bourdieu (2005), Elias y Dunning (1992), entre otros, que al analizar el rol de los deportes en la modernidad, nos permiten comprender algunos de los sentidos que se transmiten en esas prácticas, acordes al modelo social y cultural, como la competencia, la individualidad, violencia, cooperación, obediencia a reglas, entre otros. Pero ¿Qué sucede cuando estas prácticas que cargan los sentidos modernos, chocan con sentidos elaborados en otros marcos culturales? Según las observaciones en Mandari Panga, podemos decir, más que nada por sus relatos, que los sentidos que en estos se transmiten, están ligados, a los sentidos que se transmiten en las prácticas cotidianas que vinculan al juego y al trabajo. Por lo tanto, a la conservación de la identidad cultural.

Los relatos sobre los deportes en la comunidad, afirman que estas actividades se realizan con fines de integrar a otras comunidades en sus actividades y de compartir con éstas algún festín. Ya que no solo se juega al deporte, sino que alrededor de éste existen múltiples actividades que involucran a la totalidad de la 
comunidad. Por ejemplo, preparar la comida y la chicha para las comunidades invitadas, bailar, hacer música y fundamentalmente afianzar los lazos de amistad entre la comunidad y las comunidades vecinas. Por su parte las comunidades vecinas, eventualmente, en algunos acontecimientos también invitan a otras comunidades, donde son incluidos. Como me dice un comunero kichwa: "el fútbol es la única manera, para que los vecinos vengan a nuestra comunidad".

Esta experiencia, nos permite analizar que los deportes en general, están cargados de sentidos que los propios actores construimos dependiendo de nuestras experiencias culturales. En este caso, puede interpretarse que los sentidos que se transmiten en estos encuentros, están vinculados mayormente, a la unidad, la comunidad, transmisión de la propia cultura kichwa, etc., los cuales parten desde sus saberes, cosmovisiones y formas de relacionarse con el mundo.

\section{CONSIDERACIONES FINALES.}

Este trabajo intentó mostrar de manera general, visiones y saberes del cuerpo que provienen de los pueblos originarios de América Latina. Es así que a partir de las experiencias de los/as comuneros y comuneras de Mandari Panga, se ha permitido comenzar a indagar sobre la educación del cuerpo desde tal perspectiva.

Considero pertinente entonces, referirme a la importancia del territorio como punto central que engloba a la temática de la educación del cuerpo. Este es percibido como totalidad, como todo viviente, diferente a la tradición moderna que lo considera únicamente como espacio físico, sin vida (Segato, 2016). El territorio específico donde se asienta la comunidad Mandari Panga es el que permite encontrarse corporalmente con sus tradiciones kichwas y desde allí transmitir una educación, permitiéndoles aplicar los conocimientos provenientes de sus antepasados, guardados en la memoria de la comunidad para vivir en ese ambiente de una determinada manera. Esto da como resultado que la educación del cuerpo no puede ser vista únicamente desde un plano individual (como conjunto de órganos), sino que es parte de una comunidad y una idea de totalidad (naturaleza /mundo), que les da sentido a sus prácticas corporales.

De esta manera podemos afirmar que: La educación del cuerpo en Mandari Panga está vinculada principalmente a la permanencia en un tipo específico de territorio, buscado y elegido por los/as propios/as integrantes de la comunidad, con el fin de evitar el estilo de vida que se lleva a cabo en la ciudad, para poder mantener viva la tradición Kichwa. Éste, ha permitido la construcción de un saber del cuerpo, transmitido de generación en generación, que parte del conocimiento sobre la naturaleza, determinando así un tipo de alimentación, sanación del cuerpo, incluidas dentro del plano simbólico, como el chamanismo y la brujería. Las maneras de obtener los insumos para tales actividades son producto del trabajo en ese mismo medio, que se aprende desde muy joven a partir del juego. Es así que esta relación con la naturaleza y con el propio cuerpo, está mediada en gran parte por las tradiciones de los pueblos originarios que configuran a su cosmovisión, cuyo principio fundamental es el Sumak Kawsay (Buen vivir), determinando la forma de vida en comunidad y en armonía con el ambiente. A su vez, estas prácticas, son resignificadas constantemente, adoptando, debido al avance del multiculturalismo, diversas prácticas y costumbres de la cultura occidental, como son los deportes, religión, uso de distintas tecnologías, entre otras, determinando así nuevas relaciones sociales, políticas, económicas y educativas. Por esta razón es que se desea incorporar a estos saberes en participación con los demás discursos. Desde una perspectiva de totalidad del cuerpo, su educación no queda únicamente en el individuo, sino en relación a todo lo que lo rodea, tanto con la tierra como con todos los seres vivos.

Este saber es contradictorio al egoísmo y competencia que se construye en la modernidad, donde el principal sentido de existencia está ligado al aumento de la tasa de ganancia (Castoriadis, 1977). Por el contrario, estas perspectivas provenientes de pueblos originarios, nos proponen una alternativa al futuro, donde el criterio de racionalidad no prioriza el aumento económico o tecnológico, sino, lo contrario; la afirmación y crecimiento de la vida humana como totalidad. Denominados por Darcy Ribeiro (1987) como pueblos testimonios, por su relación de respeto que tienen con la naturaleza. Creando, a partir de ésta, una 
ética, economía, política, educación, que permite el postulado ecológico: la vida perpetua, que incluye a las generaciones posteriores (Dussel, 2009).

En este sentido las prácticas de la Educación Física, también se encuentran ligadas a determinados territorios, delimitados y organizados, política y económicamente a partir de relaciones de poder, configurando así una educación del cuerpo que no involucra a la totalidad de la población y restringe su acceso. La educación del cuerpo por tanto, no debe ocuparse entonces únicamente del cuerpo individual, sino de un cuerpo situado en un territorio específico, y por lo tanto, participar por la lucha de éstos con el fin de conseguir una educación de calidad (en cuanto a igualdad de posibilidades), los cuales se encuentran cada vez más controlados y privatizados (disminución de espacios públicos, clubes, accesos a la medicina, alimentación, agua, etc.), donde el derecho al juego y las prácticas educativas para el cuerpo son restringidas según la capacidad de consumo.

\section{Bibliografía.}

Bourdie, P, C. y Passeron, J, C (1996)."La reproducción" Elementos para una teoría del sistema de enseñanza. México: Fontamara S.A.

Carballo, C. (2003). Proponery negociar. Elocaso de las tradiciones autoritarias en las prácticas de la Educación Física. Un análisis del autoritarismo y de las prácticas de la Educación Física en el contexto de la Reforma Educativa (Argentina 1995-2000). La Plata: Ediciones Al Margen.

Castoriadis, C. (1977). El avance de la insignificancia" y la crisis del proceso identificatorio. En: encrucijadas del laberinto IV. Buenos Aires: Eudeba.

Citro, S. (2009). Cuerpos significantes. Travesias de una etnografía dialéctica. Buenos Aires: Biblos.

Crisorio, R, Bratch, V. (2003). La educación fisica en Argentina y en Brasil: identidad, desafios y perspectivas. Argentina: Ediciones Al Margen.

De Sousa Santos Boaventura. (2009). Una epistemología del sur. México: CLACSO y Siglo XX.

Descola, P. (2012). Más allá de la naturaleza y la cultura. Buenos Aires: Amorrortu.

Dussel, E. (1994).1492: El encubrimiento del otro. (Hacia el origen del "mito" de la modernidad). Facultad de Humanidades y Ciencias de la Educación. UMSA: Plural editores.

Dussel, E. (2007). Para una erótica Latinoamericana. Venezuela: Fundación Editorial el perro y la rana, nueva edición corregida.

Dussel, E. (2009). El pensamiento filosófico latinoamericano, del Caribe y latino (1300-2000). México: Siglo XXI.

Elias, N y Dunning, E. (1992). Deporte y ocio en el proceso de la civilización. México, Madrid, Buenos Aires: Fondo de cultura económica.

Foucault, M. (2009). Vigilar y castigar. Argentina: Siglo XXI.

Galak, E. (2014). Educación del cuerpo y política: concepciones de raza, higienismo y eugenesia en la Educación Física Argentina.Movimento: 1543-1562. Recuperado de: http://www.memoria.fahce.unlp.edu.ar/art_revistas/pr.95 74/pr.9574.pdf

Garcia Canclini, N. (1990). Culturas Hibridas (estrategias para entrar y salir de la modernidad). México D.F: Grijalbo, 1990.

Geertz, C. (1973). La interpretación de las culturas. Buenos Aires: Gedisa.

Grosfoguel, R. (2013). Racismo / sexismo epistémico, universidades occidentalizadas y los cuatro genocidios/ epistemicidios del largo siglo XIV. Bogotá, Colombia: Revista, Tabula Rasa., No.19: 31-58.Recuperado de: htt ps://www.redalyc.org/articulo.oa?id=39630036002

Huacanacuni Mamani, F. (2010). Buen Vivir / Vivir Bien. Filosofia, politicas, estrategias y experiencias regionales andinas. La paz, Bolivia: Coordinadora Andina de Organizaciones Indígenas (CAOI).

Kusch, R. (2015). Elpensamiento indígena y popular en América. Tierras del Sur: Argentina. 
Lambeck, M. (2010).Cuerpo y Mente en la Mente, Cuerpo y Mente en el Cuerpo. Algunas intervenciones antropológicas en una larga conversación. In: CITRO, Silvia (Coord.). Cuerpos Plurales: antropología de y desde los cuerpos. Buenos Aires: Biblos.

Leendhart, M. (1997). Dokamo. La persona y el mito en el mundo melanesio". Paidios.

López Austin. (1967). Cuarenta clases de magos del mundo. En: Estudios de cultura Nábuatl. Ciudad de México: UNAM. Instituto de investigaciones históricas. Volumen III.

Martínez Sarasola, C. (2014). Toda la tierra es una sola alma. Buenos Aires: Del Nuevo Extremo.

Ortiz, P. (2012). Espacio, Territorio e Interculturalidad Una aproximación a sus conflictos y resignificaciones desde la Amazonía de Pastaza en la segunda mitad del siglo XX. (Tesis doctoral en estudios culturales latinoamericanos, Universidad Andina Simón Bolívar). Recuperada de: https://www.repositorio.uasb.edu.ec/bitstream/10644/3 437/1/TD035-DECLA-Ortiz-Espacio.pdf

Pavía, V. (2006). Jugar de un modo lúdico. El juego desde la perspectiva del jugador. Buenos Aires: Noveduc.

Ribeiro, D. (1987). Configuración Histórico- Culturales Americanas. Buenos Aires: Calicanto.

Rivera Cusicanqui, S. (2010). Violencias (re) encubiertas en Bolivia. La Paz, Bolivia: Piedra Rota.

Turner, B. (1984). Paradojas del cuerpo. En: El cuerpo y la sociedad. México D.F: Fondo de cultura económica.

Segato, S. (2016). La crítica de la colonialidad en ocho ensayos (1a edición). Buenos Aires: Prometeo Libros.

Viteri Gualinga, L. (2005). Proyecto de autonomía del Pueblo Kichwa de Pastaza, región amazónica ecuatoriana. En: Pueblos indígenas, estado y democracia. Pablo Dávalos. Buenos Aires: CLACSO, Consejo Latinoamericano de Ciencias Sociales. Recuperado de: http://biblioteca.clacso.edu.ar/clacso/gt/20101026013328/16ViteriG.pdf

Wacquant, L. (2006). Entre las cuerdas. Cuadernos de un aprendiz de boxeo. Buenos Aires: Siglo XXI editores.

Willis, P. (1988): Aprendiendo a trabajar. Cómo los chicos de la clase obrera consiguen trabajos de clase obrera. Madrid. Akal.

\section{Notas}

1 Descola (2015), es uno de los autores que al estudiar a los shuaras ecuatorianos (cercanía inmediata con la comunidad estudiada) coincide en que la visión del cuerpo en estas regiones amazónicas, con las que se comparten cosmovisiones, en este caso sobre los espíritus de las plantas, animales y otros seres, son visiones del cuerpo que datan de siglos. 